\title{
Llamados a perfiles de proyectos de Innovación en el Laboratorio Tecnológico del Uruguay (LATU)
}

\author{
Castro, J. (1) \\ Contacto: jcastro@latu.org.uy \\ ${ }^{(1)}$ Gerencia de Proyectos Generales - Laboratorio Tecnológico del Uruguay (LATU)
}

\begin{abstract}
Resumen
El presente trabajo muestra las características de los llamados realizados por el Laboratorio Tecnológico del Uruguay (LATU) a PYMES y a particulares a los efectos de seleccionar Perfiles de Proyectos de Innovación. Se presenta el sistema de llamados, sus características principales y los resultados obtenidos de las tres convocatorias realizadas a partir del año 2005.

Se enfatiza en la característica de diseño y ejecución en conjunto entre el LATU y los proponentes de los Proyectos surgidos a partir de los Perfiles seleccionados.

Las conclusiones iniciales presentadas indican una buena respuesta hacia las convocatorias con beneficios para los proponentes y para la actividad del LATU.
\end{abstract}

\begin{abstract}
The paper shows the characteristics of the calls made by the Technological Laboratory of Uruguay (LATU) to SMEs and individuals, for the purpose of selecting Profiles for Innovation Projects. We present the system, its main features and the results of the three calls made since 2005.

Emphasis is made in the joint design and implementation of the projects between LATU and the proponents .

The initial findings show a good response to the calls, with mutual benefits to the proponents and LATU.
\end{abstract}

\section{Introducción}

$\mathrm{E}$ Laboratorio Tecnológico del Uruguay (LATU) es una entidad que ha estado involucrada desde su fundación en el desarrollo tecnológico del país. Su organización interna se ha dado a través de distintos sectores especializados que han evolucionado en la actual organización por departamentos que brindan apoyo técnico en diversas áreas relacionadas con la industria y la producción en general.

Se cuenta con una importante infraestructura en laboratorios y plantas piloto manejados por técnicos altamente especializados que conforman una situación muy favorable a los efectos de apoyar a las ideas innovadoras relacionadas con el desarrollo de nuevos productos y procesos tecnológicos. Además existe un importante relacionamiento con otras instituciones vinculadas con las actividades tecnológicas dentro y fuera del país.

Como parte de una política del LATU de apoyo a la innovación, en el año 2005 se entendió conveniente utilizar el mecanismo de llamado a concurso externo a los efectos de seleccionar perfiles de proyectos innovadores que serían apoyados para su desarrollo.

El presente artículo muestra las características de estos llamados y los resultados iniciales del desarrollo de los proyectos seleccionados.

\section{Características de los Ilamados}

El Programa de Desarrollo Tecnológico (PDT) y otros esfuerzos a nivel público y privado han apoyado a las empresas en el desarrollo y aplicación de tecnologías innovadoras. Estas experiencias han sido muy importantes y el LATU ha participado en diversos proyectos que han tenido financiación y apoyo por estos medios.

$\mathrm{Al}$ momento de plantearse un mecanismo propio de llamado a concurso de perfiles de proyectos de innovación, se tomó en consideración que debía apoyarse a los proponentes de ideas innovadoras no solamente en los aspectos directamente relacionados con los temas tecnológicos, sino también en la redacción del Proyecto a ejecutar que surge del Perfil seleccionado.
Por lo expuesto anteriormente, aparecen tres etapas en el proceso total:

- Etapa de presentación de los Perfiles de Proyectos, su selección por el Tribunal y comunicación a los proponentes del fallo.

- Etapa de diseño en conjunto de los Proyectos.

- Etapa de ejecución del Proyecto, evaluación y seguimiento posterior.

Se tomó como modelos básicos de convocatorias, formularios de evaluación y reglamento general los desarrollados por el PDT, adaptándolos a las especiales características de los llamados.

Los aportes que el LATU realiza en la etapa de Ejecución del Proyecto son los siguientes:

- Servicios propios del LATU (asesoramiento, análisis, uso de su infraestructura de laboratorios y plantas piloto)

- Contratación temporal de personal técnico

- Contratación de servicios técnicos y tecnológicos

- Contratación de consultorías especializadas

- Viajes de técnicos del proyecto

- Salidas de campo para recolección de muestras

- Capacitación y actualización de personal

- Participación en talleres o cursos cortos

- Eventos de promoción y divulgación

- Arrendamiento o mantenimiento de equipo de investigación o de control de calidad

- Documentación y bibliografía

- Materiales para la promoción y difusión del proyecto

- Materiales utilizados en la fabricación de prototipos y plantas piloto

- Insumos y materiales de laboratorio

- Gastos de vinculación a redes de información

- Imprevistos

El LATU no financiará:

- Obras

- Bienes de capital

- Vehículos

- Terrenos 
- Arrendamiento de inmuebles

- Salario de personal afectado a tareas de producción.

- Servicios o materiales no asociados al proyecto.

En la Figura 1 se muestra esquemáticamente el proceso global desde la convocatoria a Perfiles hasta la evaluación y seguimiento posterior del Proyecto:

Las principales características del llamado a Perfiles de Proyectos de Innovación son las siguientes:

El foco del llamado es la innovación tecnológica. Se apoya la innovación, al menos a nivel nacional, en productos, en procesos y en gestión, buscando llegar a desarrollar prototipos, formulaciones o sistemas innovadores en gestión. creación, dentro de la estructura organizativa del Laboratorio, del Departamento de Proyectos de Electrónica.

Confidencialidad. La confidencialidad de los temas de los Perfiles presentados y de los Proyectos desarrollados en conjunto está asegurada por el Laboratorio y los Reglamentos del Llamado, disponibles en la página web del LATU.

Dirección del Proyecto por parte del LATU. El Director del Proyecto es un funcionario del LATU nombrado por sus aptitudes técnicas en las tecnologías utilizadas en el Proyecto.

Ejecución financiera del Proyecto. El LATU realiza directamente la ejecución financiera del Proyecto.

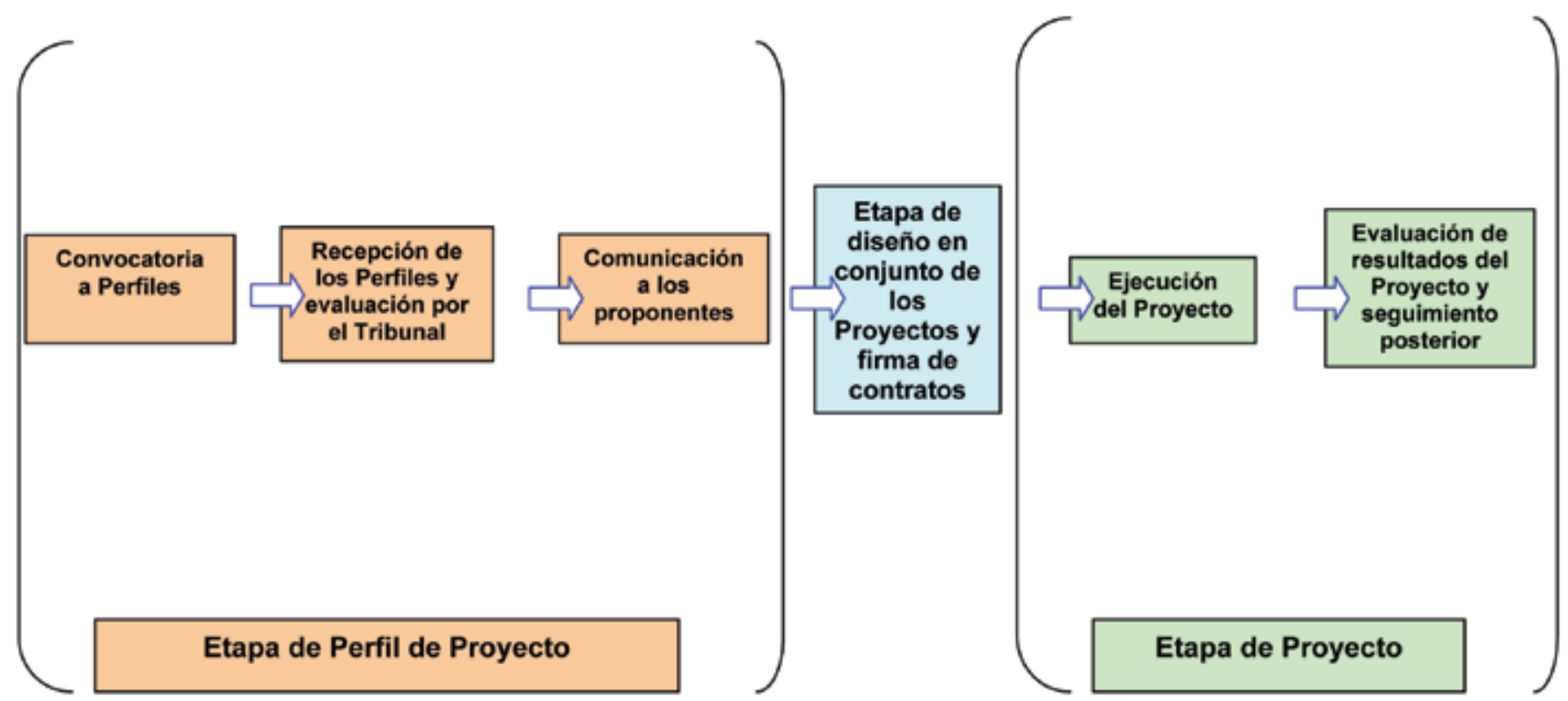

Figura 1. Esquema de proceso de llamado y selección de Perfiles, diseño del Proyecto en conjunto, ejecución y evaluación. Fuente: Elaboración propia.

Los beneficiarios directos serán PYMES y particulares con domicilio en Uruguay. Se reconoce la importancia de las PYMES en el entramado económico y social, aportando a la generación de riqueza y empleo en el país. Además se estimula la actividad innovadora de particulares que requieren apoyos para su desarrollo.

Proyecto de desarrollo en conjunto. Como instituto el LATU ha entendido que el mecanismo adecuado es el de desarrollar un Proyecto en conjunto con el proponente seleccionado. De esta manera, se realiza una convocatoria, se seleccionan los perfiles, se comunican los resultados a los proponentes y se pasa luego a una etapa de diseñar el proyecto en forma conjunta, firmar un contrato entre el proponente y el LATU y ejecutar el proyecto en cuestión.

El Tribunal está integrado por técnicos del LATU que envían a evaluar los Perfiles a técnicos especializados (pertenecientes al Laboratorio o externos), de acuerdo con la temática planteada, a los efectos de asegurar la selección de los Perfiles más innovadores.

Tecnologías. El principal aporte del LATU en estos llamados es realizado por la especialización de sus técnicos y la disponibilidad de sus recursos en materia de laboratorios y plantas piloto. Por lo tanto las tecnologías incluidas en los llamados son aquellas en que el LATU tiene fortalezas. A modo de ejemplo se puede citar que, para el llamado del año 2007 se incluyeron las siguientes tecnologías:

$\begin{array}{lll}\text { - Bioquímica } & \text { - Materiales } & \text { - Electrónica } \\ \text { - Medio Ambiente } & \text { - Energética } & \text { - Instrumentación } \\ \text { - Alimentos } & \text { - Textil } & \\ \text { - Industrial } & \text { - Ordenadores } & \end{array}$

Se han introducido en las sucesivas convocatorias nuevas tecnologías a los efectos de poner a disposición de los beneficiarios de la mayor oferta de apoyo tecnológico que el LATU puede dar. Es por esto que en 2007 se incluyó Electrónica y Ordenadores reflejando la
Difusión. La difusión del llamado se realiza por parte del LATU, mediante la publicación en su pagina web y el envío mediante correo electrónico a potenciales interesados tales como PYMES, asociaciones profesionales, instituciones del Estado, cámaras, etc.

Monto máximo por proyecto establecido en la convocatoria. Se estimula y se deja constancia en el Proyecto de los aportes de los proponentes, sin una obligación fija de aportar un porcentaje preestablecido.

Condiciones flexibles de régimen de propiedad intelectual y de uso posterior de los conocimientos generados en el Proyecto. Se estudia en cada caso particular y se negocia con el proponente dejándolo expresado en el contrato correspondiente.

Duración de los proyectos de aproximadamente un año. Se considera que esta es la duración adecuada a los efectos del planteamiento de los Perfiles.

\section{Resultados}

Desde el año 2005 se han presentado 66 Perfiles de Proyectos en las diversas tecnologías convocadas.

Se han seleccionado 20 Perfiles, estando en ejecución los Proyectos que han firmado sus contratos a la fecha de escribir este artículo.

En la Tabla 1 se puede apreciar un resumen de los resultados obtenidos en las tres ediciones de llamados realizados.

\begin{tabular}{|c|c|c|c|}
\hline Año del llamado & 2005 & 2006 & 2007 \\
\hline Perfiles presentados & 31 & 15 & 20 \\
\hline $\begin{array}{c}\text { Tecnologías en el } \\
\text { llamado }\end{array}$ & 8 & $2 *$ & 10 \\
\hline
\end{tabular}




\begin{tabular}{|c|c|c|c|}
\hline Proyectos seleccionados & 8 & 6 & 6 \\
\hline $\begin{array}{c}\text { Proyectos con contratos } \\
\text { firmados }\end{array}$ & 6 & 3 & $* *$ \\
\hline PYMES seleccionadas & 2 & 2 & 5 \\
\hline $\begin{array}{c}\text { Particulares } \\
\text { seleccionados }\end{array}$ & 4 & 1 & 1 \\
\hline
\end{tabular}

* En este año las tecnologías incluidas fueron solamente Alimentos y Energías renovables.

** Los proyectos del llamado correspondiente al año 2007 están aún en el proceso de diseño conjunto.

Tabla 1. Resultados de los llamados realizados. Fuente: Elaboración propia.

Los primeros Proyectos correspondientes al llamado de 2005 finalizarán en el correr de 2007, por lo que no se puede presentar aún los resultados de su evaluación, seguimiento y, especialmente, del posible impacto en el mercado de los desarrollos realizados.

En general se puede decir que todos los Proyectos que llegaron a la etapa de ejecución se están desarrollando normalmente con la activa participación e involucramiento de los proponentes.

\section{Conclusiones}

Cibien no se dispone de los resultados de la etapa de evaluación de los Proyectos y de su seguimiento posterior, se puede afirmar que se ha obtenido un buen nivel de convocatoria en base al número de Perfiles presentados, brindando una herramienta más a los efectos de apoyar las iniciativas innovadoras en tecnologías a nivel del país.

Esta ha sido una vía más a los efectos de estrechar la colaboración del LATU con las PYMES y particulares que desean desarrollar productos, procesos y metodologías de gestión innovadoras y que se enfrentan a carencias a nivel técnico, de infraestructura y económicos.

El interés mostrado por los beneficiarios de los Proyectos en la etapa de ejecución de los mismos demuestra el compromiso asumido y estimula a continuar con el uso y el perfeccionamiento del mecanismo de llamados.

A nivel interno del trabajo del LATU, se ha visto como una oportunidad a los efectos de captar ideas a desarrollar, con un importante aporte de los proponentes en conocimiento de los mercados y de sus demandas. Esta modalidad ha estimulado el trabajo en conjunto entre diversos departamentos técnicos y de apoyo, fortaleciendo el trabajo matricial dentro de la estructura organizativa y dando oportunidades de crecimiento profesional a los técnicos involucrados en los Proyectos.

\section{Referencias}

- DINACYT. Disponible en: www.dinacyt.gub.uy/pdt/pdt.html

- LATU. Disponible en: www.latu.org.uy 